\title{
Adam-Gibbs based model to describe the single component dynamics in miscible polymer blends under hydrostatic pressure
}

\author{
Gustavo A. Schwartz ${ }^{\text {a) }}$ \\ Centro de Fisica de Materiales CSIC-UPV/EHU, Edificio Korta, 20018 San Sebastián, Spain \\ Ángel Alegría \\ Centro de Fisica de Materiales CSIC-UPV/EHU, Edificio Korta, 20018 San Sebastián, Spain \\ and Departamento de Física de Materiales UPV/EHU, Facultad de Química, Apartado 1072, \\ 20080 San Sebastián, Spain \\ Juan Colmenero \\ Centro de Fisica de Materiales CSIC-UPV/EHU, Edificio Korta, 20018 San Sebastián, Spain; \\ Departamento de Física de Materiales UPV/EHU, Facultad de Química, Apartado 1072, \\ 20080 San Sebastián, Spain; and Donostia International Physics Center, Paseo Manuel de Lardizabal 4, \\ 20018 San Sebastián, Spain
}

(Received 6 June 2007; accepted 14 August 2007; published online 18 October 2007)

\begin{abstract}
We present in this work a new model to describe the component segmental dynamics in miscible polymers blends as a function of pressure, temperature, and composition. The model is based on a combination of the Adam-Gibbs (AG) theory and the concept of the chain connectivity. In this paper we have extended our previous approach [D. Cangialosi et al. J. Chem. Phys. 123, 144908 (2005)] to include the effects of pressure in the component dynamics of miscible polymer blends. The resulting model has been tested on poly(vinyl methyl ether) (PVME)/polystyrene (PS) blends at different concentrations and in the temperature range where the system is in equilibrium. The results show an excellent agreement between the experimental and calculated relaxation times using only one fitting parameter. Once this parameter is known the model allows calculating the size of the relevant length scale where the segmental relaxation of the dielectrically active component takes place, i.e., the so called cooperative rearrangement region (CRR) in the AG framework. Thus the size of the CRR for PVME in the blends with PS has been determined as well as its dependence with pressure, temperature, and concentration. (C) 2007 American Institute of Physics.
\end{abstract}

[DOI: $10.1063 / 1.2780157]$

\section{INTRODUCTION}

The dynamics of polymer blends has attracted the interest of many researchers during the last years because the peculiar features displayed by these systems. Among them one of the most interesting is the presence of two distinct time scales for the component segmental dynamics due to the heterogeneous dynamics at the length scale of the segmental relaxation. Several models have been proposed to describe the component segmental dynamics in miscible polymer blends. ${ }^{1-6}$ Some of these models are based on the influence of concentration fluctuations on the component dynamics. ${ }^{1,2}$ However, despite the general agreement about the ability of concentration fluctuations to affect the distribution of relaxation times, several authors have argued about their role in determining the mean relaxation time. ${ }^{3,7}$ It is now generally accepted that concentration fluctuations alone cannot account quantitatively the presence of the two distinct dynamics and the effect of chain connectivity has to be considered. ${ }^{3}$ Lodge and co-workers ${ }^{3,6}$ have used the idea that in miscible polymer blends the local concentration of one component will, on average, be richer in that component compared to the bulk

\footnotetext{
a) Author to whom correspondence should be addressed. Tel.: +34-943015389. Fax: +34-943-015600. Electronic mail: schwartz@sw.ehu.es
}

composition. This is a direct consequence of the chain connectivity. Based on these arguments and by defining the selfand effective concentration, they calculated the effective $T_{g}$ of each component in terms of the macroscopic $T_{g}$ of the neat polymers through the Fox equation. The approach of Lodge and McLeish has been later used by Leroy et al. ${ }^{4}$ who described the component segmental dynamics taking into account both the effect of concentration fluctuations and chain connectivity.

More recently, a different approach to describe the component segmental dynamics in miscible polymer blends has been proposed by us ${ }^{8,9}$ starting from the theory of Adam and Gibbs (AG) ${ }^{10}$ which relates the dynamic and thermodynamics behaviors of glass formers and has allowed us to describe the molecular dynamics close (and above) $T_{g}{ }^{11,12}$ In those works we provided a new approach to describe the component segmental dynamics of athermal miscible polymer blends (at atmospheric pressure) combining the concept of chain connectivity, expressed in terms of the selfconcentration, and the AG model. This approach has been successfully applied to different polymer blends and polymer-solvent mixtures. ${ }^{8,9,13,14}$ On the other hand, several models have been proposed in the last years to describe the pressure-temperature dependence of the relaxation time of 
neat glass forming materials. ${ }^{15,16}$ In particular, an extension of the AG theory has been recently proposed by Casalini et al. ${ }^{17}$ These authors basically proposed to write the configurational entropy in terms of the excess thermal heat capacity and of the excess thermal expansion to give account of both thermal and pressure effects, respectively. This approach has shown to be also very accurate to describe the pressure-temperature dependence of the segmental dynamics of neat polymers. ${ }^{17-21}$

The aim of this work is to present a model based on the AG theory to describe the component segmental dynamics of miscible polymer blends, at any concentration, as a function of both pressure and temperature. The idea is to combine the approach for describing the component dynamics in miscible polymer blends at atmospheric pressure ${ }^{8}$ with the pressure extension of the AG theory. ${ }^{17}$ To test the resulting model we have analyzed the segmental dynamics of poly(vinyl methylether) (PVME) on the well known PVME/polysterere (PS) system, at different concentrations. The dynamics of PVME has been widely studied under hydrostatic pressure ${ }^{20}$ as well as in binary mixtures with low molecular weight liquids ${ }^{9,22}$ and polymer blends, ${ }^{8,23,24}$ both at atmospheric and elevated pressures. $^{25-28}$ The previous works on PVME/PS blends allow us to test the accuracy of the new model in describing PVME dynamics. Furthermore, using this new approach we have also analyzed the pressure dependence of the relevant length scale for the segmental dynamics.

\section{THEORETICAL BACKGROUND}

\section{A. Adam-Gibbs theory}

The AG theory, which is based on the assumption of cooperative rearranging regions, gives an expression that relates the increase of structural relaxation time, $\tau$, to the reduction of configurational entropy, $S_{c}$, by ${ }^{10}$

$$
\tau=\tau_{0} \exp \left(\frac{C}{T S_{c}}\right),
$$

where $\tau_{0}$ is the value of $\tau$ at very high temperature and $C$ is a material constant. This quantity is here assumed independent of temperature and pressure, although this assumption has been recently questioned. ${ }^{29}$ Since $S_{c}$ is not experimentally accessible, it is usually identified with the excess entropy $\left(S_{c} \propto S_{\text {ex }}=S_{\text {melt }}-S_{\text {crystal }}\right)$. The physical meaning of this assumption is still under debate and no general agreement has been achieved, ${ }^{30-32}$ however, some findings seem to suggest a general validity of the proportionality between $S_{\mathrm{ex}}$ and $S_{c}$ which has been successfully used during the last years. Thus $S_{c}$, at atmospheric pressure, can be estimated as

$$
S_{C}(T)=g_{T} S_{\mathrm{ex}}(T)=g_{T} \int_{T_{K}}^{T} \frac{\Delta C_{P}\left(T^{\prime}\right)}{T^{\prime}} d T^{\prime},
$$

where $\Delta C_{P}=C_{P}^{\text {melt }}-C_{P}^{\text {crystal }}$ is the excess heat capacity at atmospheric pressure and $T_{K}$ is the Kauzmann temperature, where the excess entropy would vanish. If an inverse temperature dependence is assumed for $\Delta C_{P}\left(\Delta C_{P}=K / T\right)$, the empirical Vogel-Fulcher-Tammann (VFT) equation ${ }^{33} \tau(T)$ $=\tau_{0} \exp \left(D T_{0} /\left(T-T_{0}\right)\right)$ is recovered (with $\left.T_{0}=T_{K}\right)$. However, this temperature dependence for $\Delta C_{P}$ is not always valid and a linear dependence of $\Delta C_{P}$ with temperature is often a better approximation. ${ }^{34}$ If a linear dependence of the type $\Delta C_{P}$ $=b-m_{T} T$ is assumed for the temperature dependence of the excess heat capacity, then by integrating Eq. (2) and replacing in Eq. (1) we obtain the following temperature dependence for the segmental relaxation time at atmospheric pressure:

$$
\tau(T, P \cong 0)=\tau_{0} \exp \left[\frac{C / g_{T}}{T\left(b \ln \left(T / T_{K}\right)-m_{T}\left(T-T_{K}\right)\right)}\right],
$$

which in the experimentally accessible temperature range provides as good description as the VFT equation with the same number of parameters. It is worth to mention that this equation was first reported by Scherer ${ }^{35}$ in 1984 . By using Eq. (3) to fit the experimental data of neat polymers at atmospheric pressure, the three unknown parameters $\log \left(\tau_{0}[s]\right)$, $C / g_{T}$, and $T_{K}$ are obtained.

\section{B. Pressure extension of the AG theory for neat components}

Among the several models developed to describe the pressure-temperature dependence of the relaxation time of glass forming materials, ${ }^{15,16}$ a pressure extension of the AG theory has been recently proposed by Casalini et al. ${ }^{17}$ In their work they have proposed an extension of Eq. (2) for the configurational entropy $\left(S_{c}\right)$, by adding a term related to the pressure change. Thus, the temperature and pressure depen-

\begin{tabular}{|c|c|c|c|c|c|c|c|}
\hline \multirow[b]{2}{*}{ Polymer } & \multicolumn{2}{|c|}{ Thermodynamics parameters } & \multicolumn{3}{|c|}{ PVT parameters } & \multicolumn{2}{|c|}{$\begin{array}{l}\text { Structural } \\
\text { parameters }\end{array}$} \\
\hline & $\begin{array}{c}b \\
(\mathrm{~J} / \mathrm{K} \mathrm{mol})\end{array}$ & $\begin{array}{c}m_{T} \\
\left(\mathrm{~J} / \mathrm{K}^{2} \mathrm{~mol}\right)\end{array}$ & $\begin{array}{c}\Delta(\partial V / \partial T)_{P=0} \times \\
10^{2}\left(\mathrm{~cm}^{3} / \mathrm{K} \mathrm{mol}\right)\end{array}$ & $\begin{array}{c}A \times 10^{2} \\
\left(\mathrm{~cm}^{3} / \mathrm{K} \mathrm{mol}\right)\end{array}$ & $\begin{array}{c}P_{0} \\
(\mathrm{MPa})\end{array}$ & $\begin{array}{c}l_{K} \\
(\AA \AA)\end{array}$ & $\begin{array}{l}L_{P} \\
(\AA)\end{array}$ \\
\hline PVME & 68.3 & 0.160 & $3.68 \pm 0.09$ & $1.82 \pm 0.32$ & $154 \pm 22$ & 13 & 2.7 \\
\hline PS & 99.0 & 0.171 & $3.65 \pm 0.03$ & $3.02 \pm 0.01$ & $169 \pm 17$ & 18 & 3.9 \\
\hline \multicolumn{8}{|c|}{ Dynamics parameters } \\
\hline Polymer & $\log \left(\tau_{0}[s]\right)$ & $C^{\prime}(\mathrm{kJ} / \mathrm{mol})$ & $T_{K}(\mathrm{~K})$ & $g_{P} / g_{T}$ & $T_{g}(\mathrm{~K})$ & \multicolumn{2}{|c|}{$\alpha\left(\AA \mathrm{J}^{1 / 3} \mathrm{~mol}^{-1 / 3} \mathrm{~K}^{-1 / 3}\right)$} \\
\hline PVME & -13.2 & 54.81 & 200.0 & $(0.7-1.6) \times 10^{-3}\left(T-T_{g}\right)$ & 247 & \multicolumn{2}{|c|}{$10.5 \pm 1.0$} \\
\hline PS & -12.4 & 60.70 & 322.2 & $1.15 \pm 0.05$ & 373 & \multicolumn{2}{|c|}{$\ldots$} \\
\hline
\end{tabular}
dence of the $S_{c}$ is given by

TABLE I. Relevant parameters for the pure components of the here studied blends. Errors are \pm 1 of the least significant digit unless specified. 


$$
S_{c}(T, P)=g_{T} \int_{T_{K}}^{T} \frac{\Delta C_{P}\left(T^{\prime}\right)}{T^{\prime}} d T^{\prime}-g_{P}(T) \int_{0}^{P} \Delta\left(\frac{\partial V}{\partial T}\right)_{P^{\prime}} d P^{\prime},
$$

where $\Delta(\partial V / \partial T)_{P}=(\partial V / \partial T)_{P}^{\text {melt }}-(\partial V / \partial T)_{P}^{\text {crystal }}$ is the difference of the thermal expansivity of the melt and the crystal; if $g_{P}$ does not depend on temperature, then the thermal and volumetric contributions to the configurational entropy are fully decoupled. The second term in Eq. (4) gives the pressure dependence of $S_{c}$ (and therefore of the heat capacity). We have shown in previous work ${ }^{20}$ that this dependence is rather weak for PVME; these results are in agreement with those obtained by Takahara et al. ${ }^{36,37}$ on other glass formers.

It is worth noting here that $S_{\text {ex }}$ and $\Delta(\partial V / \partial T)_{P}$ are defined as the difference between melt and crystal corresponding quantities. However, it is sometimes impossible for polymers to get the thermodynamic properties of the crystalline state. Thus, it is usually assumed that the heat capacity and thermal expansion for the crystal state are similar to those found for the glass. ${ }^{38}$ Thus, we have used for calculations $\Delta C_{P} \cong C_{P}^{\text {melt }}-C_{P}^{\text {glass }} \quad$ and $\quad \Delta(\partial V / \partial T)_{P} \cong(\partial V / \partial T)_{P}^{\text {melt }}$ $-(\partial V / \partial T)_{P}^{\text {glass }}$. Additionally, the pressure dependence of $\Delta(\partial V / \partial T)_{P}$ has been empirically described according to ${ }^{20}$

$$
\Delta(\partial V / \partial T)_{P}=\Delta(\partial V / \partial T)_{P=0}-A\left[1-\exp \left(-P / P_{0}\right)\right] .
$$

Thus, by replacing Eq. (5) in Eq. (4) and integrating, the following expression for the pressure-temperature dependence of the excess entropy is obtained:

$$
\begin{aligned}
S_{\mathrm{ex}}(T, P)= & {\left[b \ln \left(\frac{T}{T_{K}}\right)-m_{T}\left(T-T_{K}\right)\right]-\frac{g_{P}}{g_{T}}\left[\Delta\left(\frac{\partial V}{\partial T}\right)_{P=0} P\right.} \\
& \left.-A\left[P-P_{0}\left(1-\exp \left(-\frac{P}{P_{0}}\right)\right)\right]\right] .
\end{aligned}
$$

Finally, by replacing Eq. (6) in Eq. (1) we obtain the pressure-temperature dependence of the segmental relaxation time for neat polymers as follows:

$$
\tau(T, P)=\tau_{0} \exp \left[\frac{C / g_{T}}{T\left\{\left[b \ln \left(T / T_{K}\right)-m_{T}\left(T-T_{K}\right)\right]-g_{P} / g_{T}\left[\Delta(\partial V / \partial T)_{P=0} P-A\left[P-P_{0}\left(1-\exp \left(-P / P_{0}\right)\right)\right]\right]\right\}}\right] .
$$

Note that most parameters of Eq. (7) can be obtained from independent experiments: by differential scanning calorimeter (DSC) $\left(b\right.$ and $\left.m_{T}\right)$, by pressure-volume-temperature $(\mathrm{PVT})\left(\Delta(\partial V / \partial T)_{P=0}, A\right.$ and $\left.P_{0}\right)$, and by the temperature dependence of the relaxation times at atmospheric pressure $\left(\log \left(\tau_{0}[s]\right), C^{\prime}=C / g_{T}\right.$, and $\left.T_{K}\right)$ through Eq. (3). In this way the only unknown parameter in Eq. (7) is the ratio $g_{P} / g_{T}$. This is the single free parameter which has to be obtained from the measurements of the relaxation time at higher pressures.

This pressure extension of the AG theory has shown to be very accurate and useful to describe the pressuretemperature dependence of the segmental relaxation time in several polymers. ${ }^{17-21}$

\section{Adam-Gibbs theory in miscible polymer blends}

We will summarize here the basic hypothesis of the model we have recently proposed to describe the segmental component dynamics in miscible polymer blends at atmospheric pressure. For a full detailed discussion, see Refs. 8 and 9.

Starting from the AG equation [Eq. (1)] we assume that for a given component in a miscible polymer blend we can write the temperature dependence of the segmental relaxation time as

$$
\tau^{A / b l e n d}(T)=\tau_{0} \exp \left[\frac{C^{\prime A / b l e n d}(T)}{T S_{\text {ex }}^{A / b l e n d}(T)}\right],
$$

where $\tau^{A / b l e n d}$ is the segmental relaxation time of component $A$ in the blend and $\tau_{0}$ is the same as defined in Eq. (1) for the same component; $C^{\prime A / b l e n d}(T)$ and $S_{\mathrm{ex}}^{A / b l e n d}(T)$ refer to regions centered around a segment of polymer $A$ and are calculated as

$$
\begin{aligned}
& S_{\mathrm{ex}}^{A / b l e n d}(T)=\phi_{\mathrm{eff}}^{A} S_{\mathrm{ex}}^{A}(T)+\left(1-\phi_{\mathrm{eff}}^{A}\right) S_{\mathrm{ex}}^{B}(T), \\
& C^{\prime A / b l e n d}(T)=\phi_{\mathrm{eff}}^{A} C^{\prime A}+\left(1-\phi_{\mathrm{eff}}^{A}\right) C^{\prime B},
\end{aligned}
$$

where $C^{\prime A / b l e n d}$ will depend on temperature if $\phi_{\text {eff }}^{A}$ does depend (see below). In these equations, which are only valid for athermal mixtures, we have used the effective concentration $\left(\phi_{\text {eff }}\right)$ to take into account the connectivity effects (see Ref. 8). At length scales of the order of few monomeric units, $\phi_{\text {eff }}$ can deviate significantly from the macroscopic concentration. $\phi_{\text {eff }}$ can be calculated as

$$
\phi_{\mathrm{eff}}=\phi_{s}+\left(1-\phi_{s}\right) \phi,
$$

where $\phi$ is the macroscopic concentration of the component under consideration and $\phi_{s}$ is the self-concentration of the same component. The self-concentration is defined as the volume fraction within a sphere of radius $r_{c}$, centered in a given monomer, occupied by the polymer chain of such a monomer. For radius $\left(r_{c}\right)$ of the relevant volume smaller than the Kuhn length, $\phi_{s}$ can be related with the excess entropy as

$$
\phi_{s}=\frac{3 l_{k} l_{p}}{2 \pi \alpha^{2}} S_{\mathrm{ex}}^{2 / 3},
$$

where $l_{P}$ is the packing length and $\alpha$ is a proportionality constant between $r_{c}$ and excess entropy ${ }^{8}$ according to

$$
r_{c}=\alpha S_{\mathrm{ex}}^{-1 / 3} .
$$


Similar equations can be written for component $B$. This set of equations [Eqs. (8)-(12)] can be exactly solved being $\alpha$ the only unknown parameter; however, we have shown in a later work ${ }^{9}$ that the parameter $\alpha$ can be determined for each polymer from an independent experiment. This model has shown to be very accurate to describe the single component dynamics in miscible polymer blends at atmospheric pressure. ${ }^{8,9,13,14}$

\section{PROPOSED MODEL}

\section{A. Component dynamics in miscible polymer blends under hydrostatic pressure}

The natural extension to describe the component segmental dynamics in miscible polymer blends under hydrostatic pressure is to combine the previous two extensions of the AG theory in a new model. By mimicking Eq. (8) we can write

$$
\tau^{A / \text { blend }}(T, P)=\tau_{0} \exp \left[\frac{C^{\prime A / \text { blend }}(T, P)}{T S_{\mathrm{ex}}^{A / \text { blend }}(T, P)}\right],
$$

where now $\tau^{4 / b l e n d}(T, P)$ is the segmental relaxation time of component $A$ in the blend as a function of pressure and temperature and $\tau_{0}$ is the same as defined in Eq. (1) also for component $A$; as previously, $C^{\prime A / b l e n d}(T, P)$ and $S_{\mathrm{ex}}^{A / \text { blend }}(T, P)$ refer to regions centered around a segment of polymer $A$ and are calculated as

$$
\begin{aligned}
& S_{\mathrm{ex}}^{A / \text { blend }}(T, P)=\phi_{\mathrm{eff}}^{A} S_{\mathrm{ex}}^{A}(T, P)+\left(1-\phi_{\mathrm{eff}}^{A}\right) S_{\mathrm{ex}}^{B}(T, P), \\
& C^{\prime A / \text { blend }}(T, P)=\phi_{\mathrm{eff}}^{A} C^{A}+\left(1-\phi_{\mathrm{eff}}^{A}\right) C^{\prime B},
\end{aligned}
$$

where $S_{\mathrm{ex}}^{A}(T, P)$ and $S_{\mathrm{ex}}^{B}(T, P)$ are the excess entropy as a function of the pressure and temperature for the neat homopolymers as defined in Eq. (6). As in the case of Eq. (9), these expressions are only valid for athermal mixtures. $\phi_{\text {eff }}$ is the effective concentration as defined in Eq. (10) with the self-concentration defined as

$$
\phi_{s}^{A / b l e n d}=\frac{3 l_{k} l_{p}}{2 \pi[\alpha(P)]^{2}}\left[S_{\mathrm{ex}}^{A / \text { blend }}(T, P)\right]^{2 / 3},
$$

where all the parameters are defined as before, being now $\alpha$ pressure dependent. This system of equations can be exactly solved once the pressure-temperature dynamics of both neat components are known, being $\alpha(P)$ the only fitting parameter for a given component.

\section{B. Implementation of the model}

In order to apply the here proposed model to a given polymer blend, it is first necessary to perform a full characterization of the pressure-temperature dynamics of both components of the blend. According to the previous section [Eqs. (6) and (7)] we have to perform DSC and PVT measurements on each of the neat components to calculated $b$ and $m_{T}$ and $\Delta(\partial V / \partial T)_{P=0}, A$, and $P_{0}$, respectively. Then, from the temperature dependence of the relaxation times at atmospheric pressure $\log \left(\tau_{0}[s]\right), C / g_{T}$, and $T_{K}$ can be obtained through Eq. (3). Finally, the only unknown parameter in Eq. (7), i.e., the ratio $g_{P} / g_{T}$, can be estimated by minimizing the mean square deviation between the experimental data and the relaxation times given by Eq. (7). This procedure gives the full temperature-pressure dependence of the segmental relaxation time for each component of the blend (see Table I).

According to Eqs. (11)-(15) we need to know the Kuhn and packing length of both components to calculate their self-concentration. In the case of PS and PVME here studied these data are available in the literature and are also listed in Table I. The remaining two parameters are the macroscopic composition of the blend $(\phi)$, which is known for each blend, and $\alpha(P)$ which is the only unknown parameter in our model and has to be estimated from the fitting of the experimental relaxation times at different temperatures and pressures.

\section{EXPERIMENT}

The experimental data used in this paper have been taken from a previous work ${ }^{28}$ where the experimental details were given and discussed. Only a brief summary is presented here.

Samples. Blends of poly(vinyl methyl ether) (PVME) $\left(\left[\mathrm{C}_{3} \mathrm{H}_{6} \mathrm{O}\right]_{n}\right) M_{w}=21900 \mathrm{~g} / \mathrm{mol}\left(M_{n}=7300 \mathrm{~g} / \mathrm{mol}\right)$ and polystyrene (PS) $\left(\left[\mathrm{C}_{8} \mathrm{H}_{8}\right]_{n}\right) \quad M_{w}=70950 \mathrm{~g} / \mathrm{mol} \quad\left(M_{n}\right.$ $=66900 \mathrm{~g} / \mathrm{mol})$ at three different concentrations were prepared. Blends with 75\%, 50\%, and 25\% in weight of PVME, namely, PVME/PS 75/25, PVME/PS 50/50, and PVME/PS $25 / 75$, are analyzed in this work.

DSC and PVT measurements. Calorimetric measurements were performed on a Q1000 TA Instruments DSC in the modulated mode, with amplitude of $0.5 \mathrm{~K}$, a period of $100 \mathrm{~s}$, and underlying cooling rate of $0.25 \mathrm{~K} / \mathrm{min}$. PVT measurements were performed on a Thermo Haake PVT100 equipment on isobaric cooling mode in the pressure range of 20-200 MPa with a cooling rate of $5 \mathrm{~K} / \mathrm{min}$.

Dielectric measurements at atmospheric pressure. Dielectric measurements were performed using a broadband dielectric spectrometer based on two high precision dielectric analyzers, one for the frequency range $10^{-2}-10^{7} \mathrm{~Hz}$ (Alpha analyzer Novocontrol $\mathrm{GmbH}$ ) and the other for the range $\left(10^{6}-1.8 \times 10^{9}\right) \mathrm{Hz}$ (Agilent 4192B), and a Novocontrol Quatro cryosystem for temperature control with a precision better than $0.1 \mathrm{~K}$.

Dielectric measurements under pressure. Dielectric measurements were carried out in a pressure cell $(0-300 \mathrm{MPa})$ supplied by Novocontrol $\mathrm{GmbH}$. The measurements were performed by frequency sweeps $\left(10^{-2}-10^{6} \mathrm{~Hz}\right)$ at constant temperature, after stabilizing the temperature of the cell for about $2 \mathrm{~h}$, with stability better than $\pm 0.1 \mathrm{~K}$, and constant pressure, with stability better than $\pm 2 \mathrm{MPa}$.

\section{RESULTS AND DISCUSSION}

\section{A. Dynamics of neat polymers}

Full DSC and PVT characterization of PVME and PS has been performed in previous works. ${ }^{8,20,21}$ The corresponding parameters are listed in Table I. Additionally, the pressure-temperature dependence of the segmental relaxation time for both neat polymers has been also analyzed in pre- 

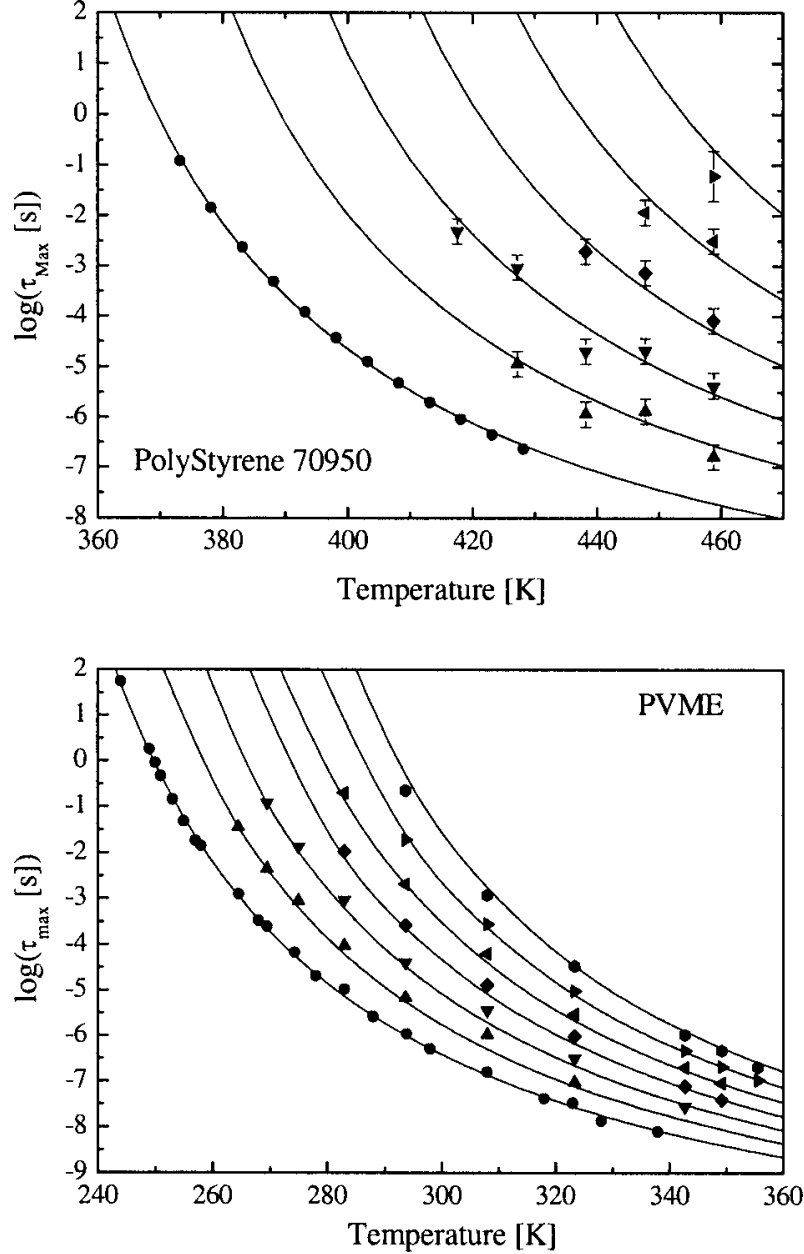

FIG. 1. Segmental relaxation time of PS and PVME as a function of the temperature at different pressures [from bottom to top: $P_{\text {atm }}=0.1,50,100$, 150, 200, and 250 (and 300 only for PVME) MPA]. The lines represent the best fit of the experimental data by means of Eq. (7) (see text).

vious works ${ }^{20,21}$ by means of Eq. (7). Figure 1 shows the temperature dependence of the maximum relaxation times for PVME and PS at several pressures. The solid lines in Fig. 1 represent the best fit of the experimental data with Eq. (7). The corresponding fitting parameters are listed in Table I.

\section{B. Dynamics of PVME in polymer blends}

Raw data of dielectric loss $\left(\varepsilon^{\prime \prime}\right)$ as a function of the frequency at constant temperature, as well as at constant pressure, for the different blend's composition are shown in Ref. 28. From those spectra the temperature dependence of the maximum relaxation time $\left(\tau_{\max }\right)$ at different pressures was obtained and is shown in Figs. 2-4. In these figures only those experimental data points within the so called equilibrium window, ${ }^{28}$ i.e., within the temperature range where the system is in thermodynamic equilibrium, are shown. It is worth to remind here that we are dealing with the dynamics of the low $T_{g}$ component of PVME/PS blends with components having very different $T_{g}$ values. Thus, by decreasing the temperature from the high temperature regime, where the whole system is at equilibrium, the high $T_{g}$ component starts freezing first and the system begins falling out of equilibrium whereas the other component is still moving relatively fast.

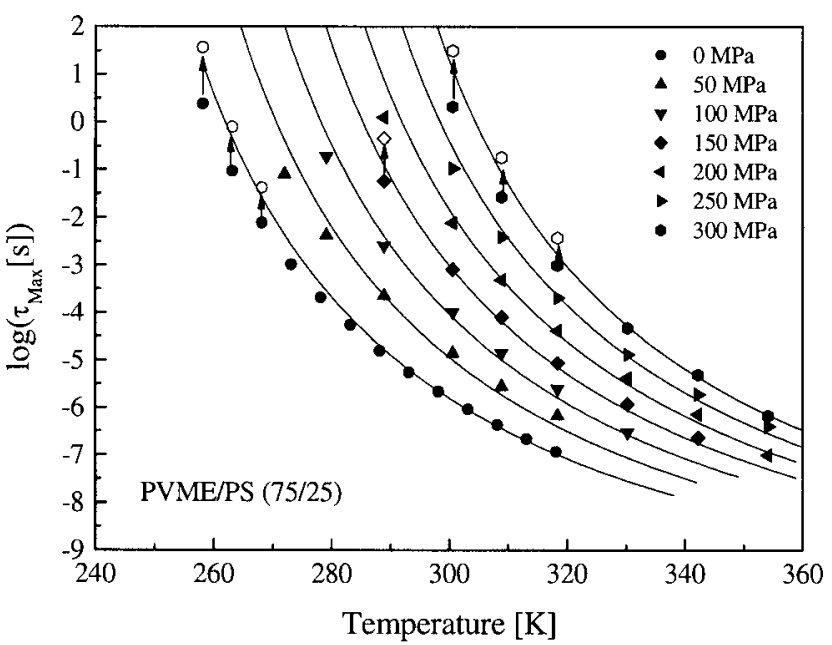

FIG. 2. Logarithm of the relaxation time of PVME vs temperature at different pressures (from bottom to top: $P_{\text {atm }}=0.1,50,100,150,200,250$, and $300 \mathrm{MPa})$ for PVME/PS (75/25). The filled symbols represent the experimental maximum relaxation times, whereas the open ones represent the estimated relaxation time of the average concentration (see text). The solid lines represent the fit of the experimental data with the here proposed model.

In this low temperature range the low $T_{g}$ dynamics is affected by the confinement imposed by the other component. We know from a previous work ${ }^{8}$ that the lower limit of the equilibrium window $\left(T_{\text {eq }}\right)$, at atmospheric pressure, is approximately 280,300 , and $330 \mathrm{~K}$ for $75 \%, 50 \%$, and $25 \%$ of PVME in the blend. Since the here presented model, in its current form, is limited to describe the relaxation dynamics only at equilibrium conditions, the low temperature range, i.e., below the equilibrium window, has not been considered in this work.

Figures 2-4 show the comparison between the experimental data and the calculated relaxation times, using the here proposed model, for PVME in blends with $75 \%, 50 \%$, and $25 \%$ of this component as a function of the temperature at different pressures. The only fitting parameter for each

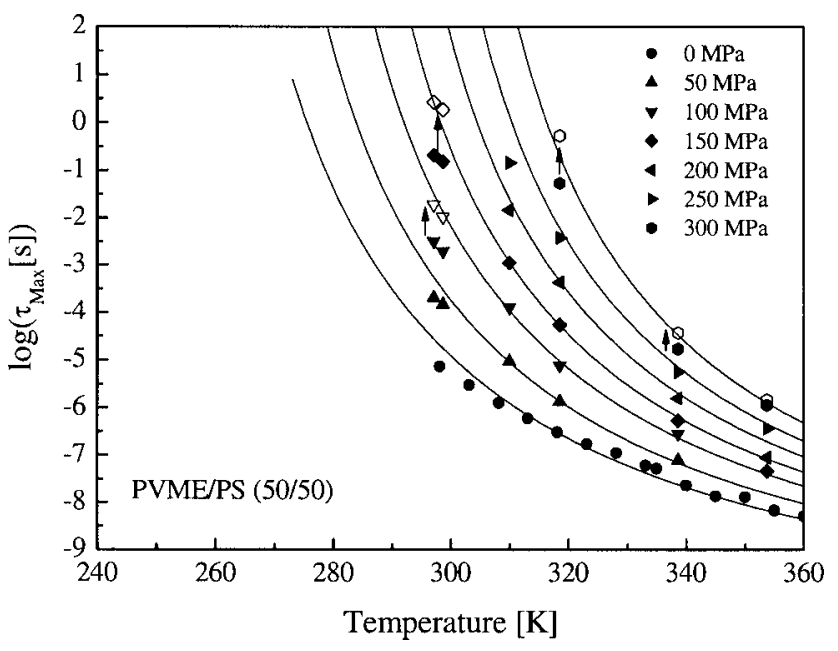

FIG. 3. Logarithm of the relaxation time of PVME vs temperature at different pressures (from bottom to top: $P_{\text {atm }}=0.1,50,100,150,200,250$, and $300 \mathrm{MPa})$ for PVME/PS (50/50). The filled symbols represent the experimental maximum relaxation times, whereas the open ones represent the estimated relaxation time of the average concentration (see text). The solid lines represent the fit of the experimental data with the here proposed model. 


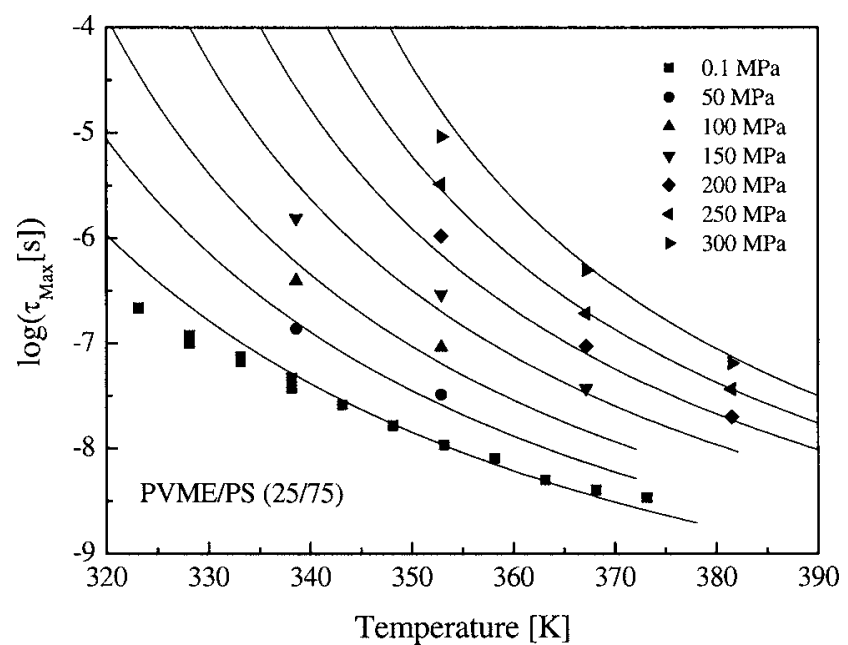

FIG. 4. Logarithm of the relaxation time of PVME vs temperature at different pressures (from bottom to top: $P_{\mathrm{atm}}=0.1,50,100,150,200,250$, and $300 \mathrm{MPa})$ for PVME/PS (25/75). The filled symbols represent the experimental maximum relaxation time. The solid lines represent the fit of the experimental data with the here proposed model.

individual curve, $\alpha(P)$, has been determined at each pressure and composition in order to fit the high temperature data range where the possible effects of concentration fluctuations are less relevant (see discussion below). The values of the $\alpha$ parameter as a function of pressure at different concentrations are shown in Fig. 5. It can be observed that $\alpha$ decreases almost linearly with increasing pressure for the three compositions. The slope of this linear dependence is, within experimental uncertainties, hardly dependent of the blend composition as shown in Fig. 5. This fact opens the possibility of calculating $\alpha(P)$ just for one composition.

Figures $2-4$ show that at low temperatures the calculated relaxation times tend to be systematically slower than the experimental maximum relaxation times. This fact is not an evidence of the limitations of the model but an effect which can be rationalized by the presence of the statistical thermal concentration fluctuations which yield a local heterogeneous distribution of both components in the blend. Since the di-

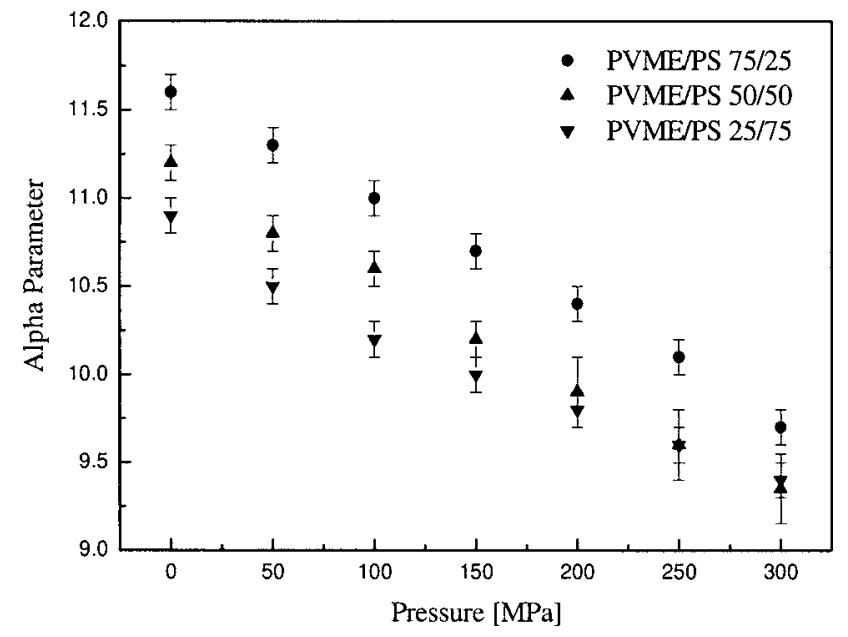

FIG. 5. Parameter $\alpha$ as a function of pressure for the three different concentrations: (75/25) circles, (50/50) upper triangles, and (25/75) down triangles. electric technique enhances those regions richer in the dielectrically active component (PVME), due to its higher contribution to the dielectric signal, the resulting relaxation time for such regions is shorter than the corresponding for regions with average (or even lower than) macroscopic concentration. Thus, the main contribution to the dielectric loss peak comes from regions richer in PVME giving an effective maximum relaxation time shorter than that predicted by the model, which would correspond to an average concentration. This is especially important at temperatures close to $T_{g}$ where concentration fluctuations originate the dramatic relaxation broadening experimentally observed for PVME in blends with PS, ${ }^{4}$ and therefore the relaxation time of the average concentration can be significantly slower than that obtained from the dielectric peak maximum. Contrary, at higher temperatures the concentration fluctuations do not play a major role in determining the shape of the segmental dynamics relaxation since it becomes close to that of the pure polymer. Therefore, in this high temperature range, maximum relaxation times obtained from dielectric spectra are found to be very close to those corresponding to the average concentration.

In order to test this hypothesis we have made an estimation of the relaxation time characteristic of the mean concentration. Based on previous results ${ }^{4}$ we have used the distribution of effective concentrations, accounting for the statistical thermal concentration fluctuations, to calculate the dielectric response in the blends with $75 \%$ and $50 \%$ of PVME and hence found the corresponding PVME relaxation time for the mean concentration. The so obtained values for some representative pressures and temperatures (at relatively long times) are shown as open symbols in Figs. 2 and 3. These new relaxation times show that the model gives an excellent description of the PVME relaxation time for the mean concentration. This also shows the importance of the statistical thermal concentration fluctuations in the relaxation behavior close to $T_{g}$. Unfortunately, for the blend with $25 \%$ of PVME the distribution function of the effective concentrations is not available and therefore we cannot perform the same estimation.

These results evidence the difficulties in developing a complete and quantitative model of the component segmental dynamics in athermal miscible polymer blends. However, the proposed model gives a very good description of the component dynamics over a broad range of pressures, temperatures, and concentrations with just only one fitting parameter $(\alpha(P))$. At atmospheric pressure it has been shown that the parameter $\alpha$ can be determined from an independent experiment and thus the model becomes completely predictive. It is expected that a similar independent determination of the parameter $\alpha(P)$ at higher pressures can be done.

\section{Pressure-temperature dependence of the size of the CRR}

As previously mentioned the AG theory allows calculation of the temperature and pressure dependence of the size of the relevant volume for the segmental relaxation (CRR) by means of Eq. (12). Thus, once the excess entropy and $\alpha$ 


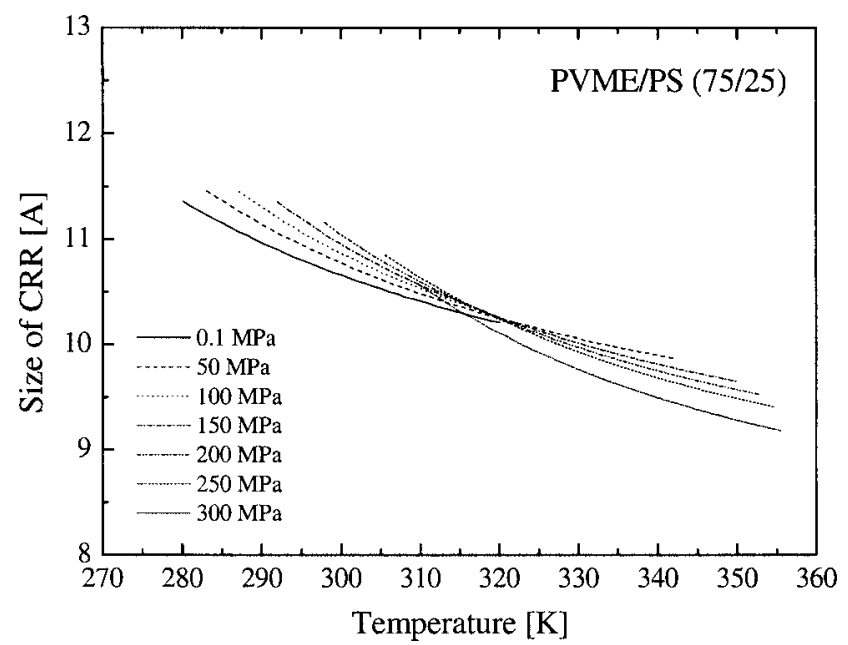

FIG. 6. Diameter of the relevant volume for segmental relaxation vs temperature, at different pressures, as calculated using the model for PVME in blend PVME/PS (75/25).

parameter are known, Eq. (12) can be used to estimate the size $\left(2 r_{c}\right)$ of the CRR at any temperature, pressure, and composition. Figures 6-8 show the temperature dependence, at different pressures, of the size of the CRR for PVME in PVME/PS (75/25), (50/50), and (25/75) blends, respectively. The size of the CRR is around $1 \mathrm{~nm}$, in agreement with previous results at atmospheric pressure on the same blend. $^{8}$ The increasing length upon temperature reduction imposed by the AG theory is clearly observed in these figures although less pronounced for $(25 / 75)$ blend because the narrower temperature range experimentally available. As regards the effects of pressure, a slight but systematic increment of the temperature sensitivity of the CRR's size with pressure is observed for the three concentrations. This is very clear for the 50/50 blend where the effects of pressure are more evident. This fact is probably due to differences in the monomeric volume of both polymers and its effects on local packing which has shown to play a key role in controlling the dynamics of glass formers. ${ }^{39}$ However, the local packing

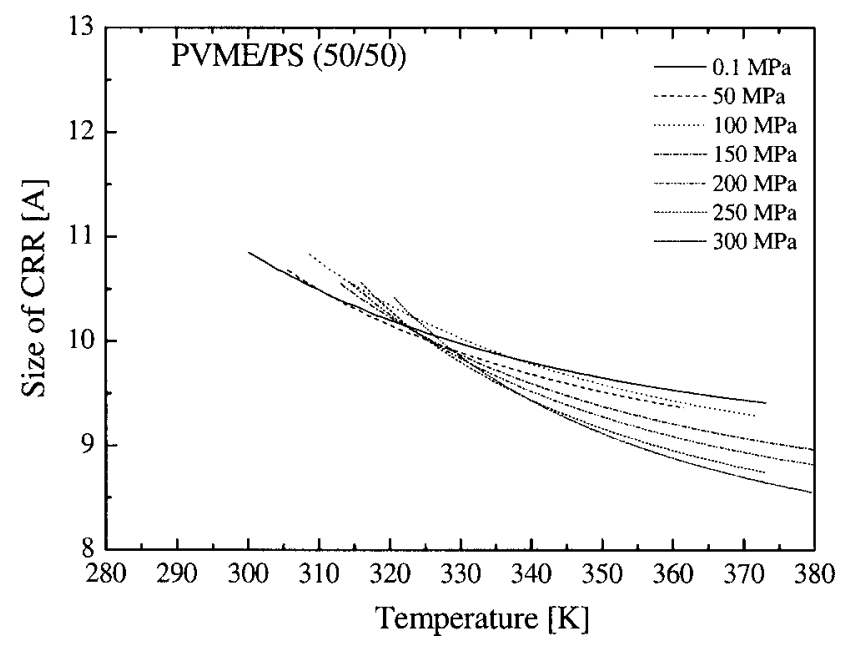

FIG. 7. Diameter of the relevant volume for segmental relaxation vs temperature, at different pressures, as calculated using the model for PVME in blend PVME/PS (50/50).

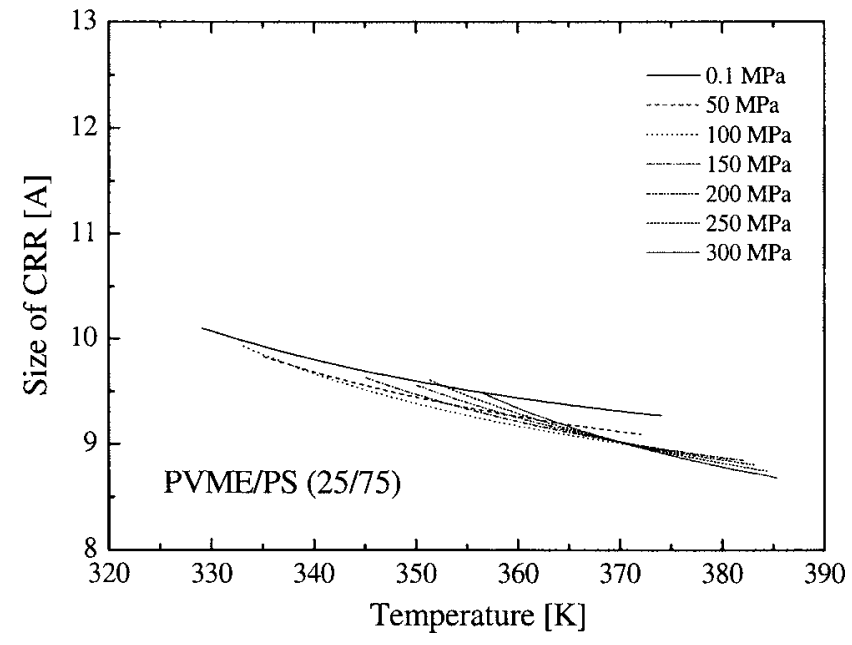

FIG. 8. Diameter of the relevant volume for segmental relaxation vs temperature, at different pressures, as calculated using the model for PVME in blend PVME/PS (25/75).

of polymer blends and its effects on the blend dynamics are beyond the scope of the present study and are worth to be analyzed in future works.

\section{CONCLUSIONS}

We have presented a model based on the AG theory to describe the segmental component dynamics in miscible polymer blends as a function of pressure, temperature, and concentration. A very good agreement between calculated and experimental relaxation times has been shown. Based on the knowledge of the pressure-temperature dependence of the dynamics of the neat polymers, the model gives account of the component dynamics in the blend by means of only one fitting parameter $(\alpha)$. This parameter is characteristic of a given component and it is expected, as in the case of atmospheric pressure, that it can be determined by means of independent experiments. If so, the here presented model would become completely predictive, once the relevance of the statistical thermal concentration fluctuations on the dielectric relaxation time are considered. Additionally, we were able to evaluate the size of the relevant length scale (CRR) where the relaxation process takes place as well as its temperature, pressure, and composition dependence. Our results show a decreasing CRR size with increasing dilution, temperature, or pressure with values between 0.8 and $1.2 \mathrm{~nm}$ in agreement with previous results.

\section{ACKNOWLEDGMENTS}

This work has been supported in part by the Spanish Ministry of Science and Technology (MICyT) (Project No. MAT 2004/01617) and by the Government of the Basque Country (Project No. 9/UPV 00206 215-13568/2001). The authors want also to thank the support of the SoftComp NoE/ NMP3-CT-2004-502235.

${ }^{1}$ G. Katana, E. W. Fischer, Th. Hack, V. Abetz, and F. Kremer, Macromolecules 28, 2714 (1995).

${ }^{2}$ S. K. Kumar, R. H. Colby, S. H. Anastasiadis, and G. Fytas, J. Chem. Phys. 105, 3777 (1996). 
${ }^{3}$ T. P. Lodge and T. C. B. McLeish, Macromolecules 33, 5278 (2000).

${ }^{4}$ E. Leroy, A. Alegría, and J. Colmenero, Macromolecules 36, 7280 (2003).

${ }^{5}$ C. M. Roland and K. L. Ngai, Macromolecules 25, 5765 (1992).

${ }^{6}$ J. C. Haley, T. P. Lodge, Y. He, M. D. Ediger, E. D. von Meerwall, and J. Mijovic, Macromolecules 36, 6142 (2003).

${ }^{7}$ T. R. Lutz, Y. He, M. D. Ediger, M. Pitsikalis, and N. Hadjichristidis, Macromolecules 37, 6440 (2004).

${ }^{8}$ D. Cangialosi, G. A. Schwartz, A. Alegría, and J. Colmenero, J. Chem. Phys. 123, 144908 (2005).

${ }^{9}$ G. A. Schwartz, D. Cangialosi, A. Alegría, and J. Colmenero, J. Chem. Phys. 124, 154904 (2006).

${ }^{10}$ G. Adam and J. H. Gibbs, J. Chem. Phys. 28, 139 (1965).

${ }^{11}$ R. Richert and C. A. Angell, J. Chem. Phys. 108, 9016 (1998).

${ }^{12}$ D. Cangialosi, A. Alegría, and J. Colmenero, Europhys. Lett. 70, 614 (2005).

${ }^{13}$ D. Cangialosi, A. Alegría, and J. Colmenero, Macromolecules 39, 448 (2006).

${ }^{14}$ D. Cangialosi, A. Alegría, and J. Colmenero, Macromolecules 39, 7149 (2006).

${ }^{15}$ N. W. Tschoegl, W. G. Knauss, and I. Emri, Mech. Time-Depend. Mater. 6, 53 (2002).

${ }^{16}$ I. Avramov, J. Non-Cryst. Solids 351, 3163 (2005).

${ }^{17}$ R. Casalini, S. Capaccioli, M. Lucchesi, P. A. Rolla, and S. Corezzi, Phys. Rev. E 63, 031207 (2001).

${ }^{18}$ D. Prevosto, M. Lucchesi, S. Capaccioli, R. Casalini, and P. A. Rolla, Phys. Rev. B 67, 174202 (2003); D. Prevosto, S. Capaccioli, M. Lucchesi, and P. Rolla, J. Non-Cryst. Solids 351, 2611 (2005).

${ }^{19}$ G. A. Schwartz, E. Tellechea, J. Colmenero, and A. Alegría, J. NonCryst. Solids 351, 2616 (2005).

${ }^{20}$ G. A. Schwartz, J. Colmenero, and A. Alegría, Macromolecules 39, 3931 (2006).
${ }^{21}$ G. A. Schwartz, J. Colmenero, and A. Alegría, J. Non-Cryst. Solids (in press).

${ }^{22}$ S. Cerveny, J. Colmenero, and A. Alegría, Macromolecules 38, 7056 (2005).

${ }^{23}$ A. Alegría, D. Gomez, and J. Colmenero, Macromolecules 35, 2030 (2002).

${ }^{24}$ J. A. Pathak, R. H. Colby, G. Floudas, and R. Jerome, Macromolecules 32, 2553 (1999) and references therein.

${ }^{25}$ G. Floudas, in Broadband Dielectric Spectroscopy, edited by F. Kremer and A. Schonhals, (Springer-Verlag, Berlin, 2003), Chap. 8.

${ }^{26}$ C. M. Roland and R. Casalini, Macromolecules 38, 8729 (2005).

${ }^{27}$ C. M. Roland, K. J. McGrath, and R. Casalini, Macromolecules 39, 3581 (2006).

${ }^{28}$ G. A. Schwartz, J. Colmenero, and A. Alegría, Macromolecules 40, 3246 (2007).

${ }^{29}$ G. P. Johari, J. Chem. Phys. 119, 635 (2003).

${ }^{30}$ C. A. Angell and S. Borick, J. Non-Cryst. Solids 307-310, 393 (2002) and references therein.

${ }^{31}$ G. P. Johari, J. Non-Cryst. Solids 307-310, 387 (2002).

${ }^{32}$ G. P. Johari, J. Chem. Phys. 116, 2043 (2002)

${ }^{33}$ H. Vogel, Phys. Z. 22, 645 (1921); G. S. Fulcher, J. Am. Ceram. Soc. 8, 339 (1923); G. Tammann and W. Z. Hesse, Z. Anorg. Allg. Chem. 156, 245 (1926).

${ }^{34}$ I. M. Hodge, Macromolecules 20, 2897 (1987).

${ }^{35}$ G. W. Scherer, J. Am. Ceram. Soc. 67, 504 (1984).

${ }^{36}$ S. Takahara, O. Yamamuro, and H. Suga, J. Non-Cryst. Solids 171, 259 (1994).

${ }^{37}$ S. Takahara, M. Ishikawa, O. Yamamuro, and T. Matsuo, J. Phys. Chem. B 103, 792 (1999).

${ }^{38}$ O. Yamamuro, I. Tsukushi, A. lindqvist, S. Takahara, M. Ishikawa, and T. Matsuo, J. Phys. Chem. B 102, 1605 (1998).

${ }^{39}$ G. Floudas, K. Mpoukouvalas, and P. Papadopoulos, J. Chem. Phys. 124, 074905 (2006). 